\title{
Coronary Atherosclerosis T1-weighed Characterization with integrated anatomical reference (CATCH)
}

\author{
Yibin Xie ${ }^{1 *}$, Jianing Pang ${ }^{1}$, Young Jin Kim², Qi Yang ${ }^{1}$, Jung-Sun Kim², Christopher T Nguyen ${ }^{1}$, Byoung Wook Choi ${ }^{2}$, \\ Zhaoyang Fan', Daniel S Berman'1, Hyuk-Jae Chang², Debiao Li ${ }^{1}$
}

From 19th Annual SCMR Scientific Sessions

Los Angeles, CA, USA. 27-30 January 2016

\section{Background}

The detection of high-risk coronary atherosclerotic lesions before severe plaque complications is the "holy grail" in cardiology. Recently T1-weighted (T1w) MRI with [2] or without [3] contrast enhancement (CE) has been used for characterizing coronary plaques showing promising prognostic value [4]. However the drawbacks of current protocols based on conventional Cartesian acquisition and navigator gating hinder the clinical application of this technique: a) coverage is limited to proximal coronary segments; b) spatial resolution is low and often anisotropic; c) because normal tissue in T1w images is highly suppressed, a separate MRA acquisition is needed to provide anatomical reference. The purpose of this work is to develop a highly accelerated MR technique for coronary plaque characterization with 1) whole-heart coverage, 2) fine isotropic spatial resolution, and 3 ) simultaneously acquired brightblood anatomical reference.

\section{Methods}

CATCH consists of ECG-gated, inversion recovery (IR) prepared spoiled gradient echo sequence with golden angle 3D radial trajectory to acquire dark-blood $\mathrm{T} 1 \mathrm{w}$ images and bright-blood reference images in an interleaved fashion (Fig. 1). Retrospective motion correction with $100 \%$ respiratory gating efficiency was performed as described previously [5]. Healthy volunteers $(n=12)$ and CAD patients with stable and unstable angina $(\mathrm{n}=$ 26) were scanned on a $3 \mathrm{~T}$ scanner (Siemens Magnetom Trio) before and after CE. Scan parameters: whole-heart
3D slab with FOV $=330^{3} \mathrm{~mm}^{3}$; spatial resolution $=1.1^{3}$ $\mathrm{mm}^{3}$; TR/TE $=4.6 / 2.3 \mathrm{~ms}$; number of radial projections $=8500$; scan time $=\sim 10$ minutes depending on heart rate. After completing MRI, 21 CAD patients further underwent interventional X-ray angiography (XA) and intracoronary optical coherence tomography (OCT) for coronary plaque evaluation. OCT images were graded for high-risk coronary plaque features (lipid-richness, macrophages, microvessels, cholesterol crystals) by two experienced cardiologists without the knowledge of MR results.

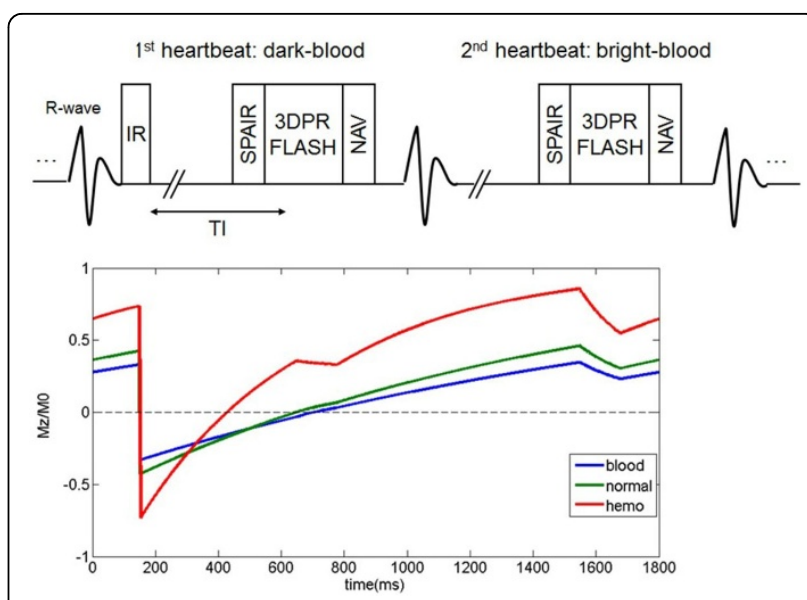

Figure 1 Upper panel: Sequence diagram of CATCH. Lower panel: Simulated steady-state signal behavior of blood, normal vessel wall, and intra-plaque hemorrhage.

${ }^{1}$ Cedars-Sinai Medical Center, Los Angeles, CA, USA

Full list of author information is available at the end of the article 


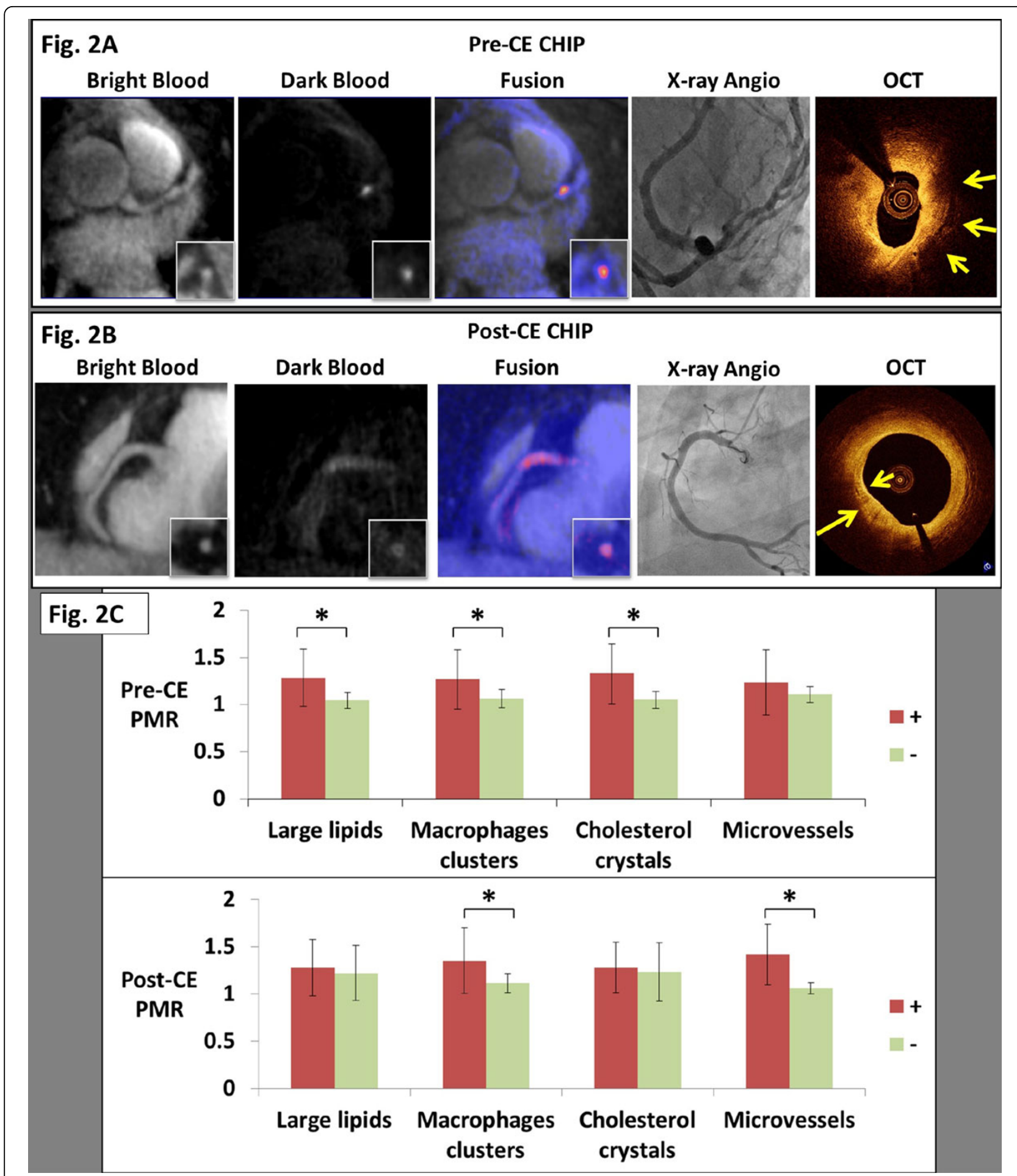

Figure 2 A: An example of pre-CE CHIP was found at middle LAD as localized on the bright-blood images. XA showed significant stenosis (70\%) at that location. OCT showed large signal-poor area suggestive of possible lipid core and/or intra-plaque hemorrhage (yellow arrow). B: An example of post-CE CHIP with diffuse wall enhancement at proximal RCA as localized on the bright-blood images. XA showed only mild stenosis (30\%) at that location. OCT showed strong multi-focal back reflections and signal heterogeneity within the overlaying tissue suggestive of high macrophage density (yellow arrows). C: Coronary plaques with high-risk features as classified by OCT tended to be hyperintensive on CATCH images. Star signs $\left(^{*}\right)$ denote statistical significance $(p<0.05)$. Positive sign $(+)$ and negative sign $(-)$ denote lesion groups with corresponding OCT grading. Plaque hyper-intensity is presented in terms of plaque to myocardium ratio (PMR) as described previously [4]. 


\section{Results}

All 38 subjects successful completed the pre-CE exams. All 12 healthy volunteers and 23 eligible patients also completed the post-CE exams. None of the healthy subjects showed coronary hyper-intensive plaques (CHIPs) in either pre-CE or post-CE T1w MRI. In total 3 patients showed CHIPs on pre-CE exams and 4 patients showed CHIPs on post-CE exams, respectively. Fig. 2A and Fig. $2 \mathrm{~B}$ are two representative patient cases with a pre-CE CHIP and a post-CE CHIP, respectively, with corresponding imaging evidences from other modalities. Fig. $2 \mathrm{C}$ is the lesion-based statistics showing elevated plaque hyper-intensity in the advanced lesions as classified by OCT.

\section{Conclusions}

The proposed MR technique of accelerated T1w whole heart coronary plaque characterization with simultaneously acquired anatomical reference was feasible. Coronary plaque hyper-intensity showed positive association with certain high-risk plaque features on OCT.

\section{Authors' details}

${ }^{1}$ Cedars-Sinai Medical Center, Los Angeles, CA, USA. ${ }^{2}$ Yonsei University

College of Medicine, Seoul, Korea (the Republic of).

Published: 27 January 2016

doi:10.1186/1532-429X-18-S1-O22

Cite this article as: Xie et al:: Coronary Atherosclerosis T1-weighed

Characterization with integrated anatomical reference (CATCH). Journal of Cardiovascular Magnetic Resonance 2016 18(Suppl 1):O22.

Submit your next manuscript to BioMed Central and take full advantage of:

- Convenient online submission

- Thorough peer review

- No space constraints or color figure charges

- Immediate publication on acceptance

- Inclusion in PubMed, CAS, Scopus and Google Scholar

- Research which is freely available for redistribution

Submit your manuscript at www.biomedcentral.com/submit 